\title{
Estágio curricular supervisionado: dificuldades e perspectivas vivenciadas por acadêmicos de enfermagem
}

\author{
Supervised curricular stage: difficulties and perspectives enhanced by academics of \\ nursing
}

Estadio curricular supervisado: dificultades y perspectivas vivenciadas por académicos de enfermería

Livia Maria da Silva ${ }^{1 *}$, Tuanny Caroline Pereira de Santana ${ }^{1}$, Laise Risalva Farias Gouveia da Silva ${ }^{1}$, Leticia Monteiro Rocha ${ }^{1}$, Camilla Talita Silva Canhoto ${ }^{1}$, Karoline Lima Dantas ${ }^{1}$, Ana Cristina Farah Abdon da Silva ${ }^{1}$, Eliana Valetim da Silva ${ }^{1}$, Maria Inês Bezerra de Melo ${ }^{1}$, Reneide Muniz da Silva ${ }^{1}$, Maria Cristina dos Santos Figueira', Rutheanne Melo Siqueira ${ }^{1}$, Mirella Raquel Romão Martins ${ }^{1}$, Suzana Lins da Silva ${ }^{1}$, Carina Ribeiro de Oliveira ${ }^{1}$.

\section{RESUMO}

Objetivo: Descrever as principais dificuldades e possibilidades vivenciadas pelos acadêmicos de enfermagem no exercício do estágio curricular supervisionado nas unidades de saúde. Métodos: Trata-se de uma revisão integrativa com abordagem quantitativa e qualitativa. Resultados: Os dados obtidos na pesquisa mostram que a principal vantagem do estágio supervisionado foi à aquisição e o aprimoramento de conhecimentos e habilidades, porém, a maioria dos estudantes encontraram algumas dificuldades, relacionadas ao distanciamento entre a metodologia teórica e prática, associado a experiência do estágio ser insuficiente para formação profissional. Conclusão: Embora seja visível os desafios enfrentados pelos acadêmicos de enfermagem durante sua vivência frente ao estágio supervisionado, esse momento é promissor de experiências profissionais, as quais visam fortalecer o processo de ensino-aprendizagem. Entretanto, há necessidade de propostas voltadas à melhoria do referido estágio.

Palavras-chave: Educação Baseada em Competências, Educação em Enfermagem, Enfermagem, Estágio Clínico.

\begin{abstract}
Objective: To describe the main difficulties and possibilities experienced by nursing students in supervised curricular training. Methods: This is an integrative review, with a quantitative and qualitative approach. Results: The data obtained in the research show that the main advantage of the supervised stage was the acquisition and improvement of knowledge, however, most of the students encountered some difficulties related to the distance between the theoretical and practical methodology, associated with the experience of the stage being insufficient for vocational training. Conclusion: Although the challenges faced by nursing students during their experience in the supervised stage are visible, this moment is promising of professional experiences aimed at strengthening the teaching-learning process. However, there is a need for proposals aimed at improving this stage.
\end{abstract}

Key words: Competency-Based Education, Education, Nursing, Clinical Clerkship.

${ }^{1}$ Faculdade Pernambucana de Saúde (FPS), Recife/PE. *E-mail: liams@hotmail.com.br

SUBMETIDO EM: 3/2019

ACEITO EM: 4/2019

PUBLICADO EM: 7/2019

REAS/EJCH | Vol .Sup.18 | e662 | DOI: https://doi.org/10.25248/reas.e662.2019 Página 1 de 10 


\section{RESUMEN}

Objetivo: Describir las principales dificultades y posibilidades vivenciadas por los académicos de enfermería en el ejercicio del estadio curricular supervisado en las unidades de salud. Métodos: Se trata de una revisión integrativa con abordaje cuantitativo y cualitativo. Resultados: Los datos obtenidos en la investigación muestran que la principal ventaja de la etapa supervisada fue a la adquisición y el perfeccionamiento de conocimientos y habilidades, sin embargo, la mayoría de los estudiantes encontraron algunas dificultades relacionadas al distanciamiento entre la metodología teórica y práctica, asociado a la experiencia de la práctica ser insuficiente para la formación profesional. Conclusión: Aunque sea visible los desafíos enfrentados por los académicos de enfermería durante su vivencia frente a la etapa supervisada, ese momento es prometedor de experiencias profesionales que apuntan a fortalecer el proceso de enseñanzaaprendizaje. Además, hay necesidad de propuestas dirigidas a mejorar dicha práctica.

Palavras-clave: Educación Basada en Competencias, Educación en Enfermería, Enfermería, Etapa Clínica.

\section{INTRODUÇÃO}

O estágio acadêmico de enfermagem é considerado o momento de aprendizagem essencial para o discente, em virtude de proporcionar não somente uma vasta experiência no âmbito acadêmico, capacitandoo para prestar o cuidado integral à saúde do paciente, como também, por oferecer um amplo crescimento pessoal e profissional. Não devendo restringir apenas aos princípios teóricos, mas também confrontar o conhecimento adquirido pelo aluno as práticas assistenciais em seu ambiente de atuação, através da interdisciplinaridade e a integração entre ensino-serviço-comunidade (LIMA TC, 2014).

Sabe-se que o estágio supervisionado, traz ao discente não somente a ampliação de seus saberes, enfatizando a associação teórico-prático, mas também contribui para o desenvolvimento de competências transversais (responsabilidade, autonomia e segurança) ao acadêmico no último ano do curso de graduação e início da sua trajetória profissional e com isso oportuniza uma maior empregabilidade dos recém-formados (EVANGELISTA DL, 2014; RIGOBELLO JL, et al., 2018).

Considerando a necessidade de uma base jurídica-legal, o ministério da educação criou uma Resolução no 3/2001 das Diretrizes Curriculares (DNC) voltada a essa atividade de ensino, o Estágio Curricular Supervisionado na graduação de enfermagem. Tal resolução contempla, não somente, a obrigatoriedade dessa modalidade de ensino, como também, é composta por uma lista de competências e habilidades profissionais, tais como, atenção à saúde, tomada de decisão, comunicação, liderança, administração/gerenciamento e educação permanente (MINISTÉRIO DA EDUCAÇÃO BR, 2001).

Diante desse aspecto legal, o currículo de enfermagem na graduação é comumente estruturado em cinco anos, com disciplinas organizadas em crescentes níveis de complexidade que objetivam formar um enfermeiro generalista que possa desenvolver a assistência de enfermagem em áreas diversificadas, buscando atender as exigências do mercado profissional, além de formar alunos com competência técnica, científica, administrativa e política (SILVA CM, 2013).

A maioria dos acadêmicos de enfermagem e porque não dizer uma grande parte, idealizam diversas formas de "competência profissional" como ferramenta para a construção do futuro enfermeiro. E isso traz uma preocupação a mais pela busca incessante por qualidade na prestação dos serviços de saúde, bem como, do seu papel clínico inovador enquanto sujeito de uma equipe multiprofissional. No entanto, ao se depararem com a rotina arcaica, desatualizada das instituições de saúde, muitos dos sonhos idealizados durante o curso de graduação são engessados pelos serviços institucionalizados, levando a estagnação de seus projetos de crescimento profissional (SOUZA FA; PAIANO M, 2011).

Segundo Alves EA (2014), no período de formação profissional os estudantes de enfermagem vivenciam as primeiras práticas curriculares, tanto na atenção básica, como na área hospitalar, entrando em contato com a realidade da saúde brasileira. Posto que a área de saúde lida com complexas situações e 
necessidades; que exigem do profissional a aplicação de competências clínicas profissionais, cujo desenvolvimento possui ligação intrínseca com o processo educativo, o mundo do trabalho e da vida (BENITO GA; TRISTÃO KM, et al., 2012). A maneira mais eficaz de adquirir uma assessoria técnico-científico voltada ao conhecimento de competências baseadas em evidências científicas e habilidades clínicas na área de saúde é através de uma educação permanente, ao exercitar ações que exijam a interação entre os domínios cognitivos e técnicos, ou seja, aprender executando tarefas, ou construindo-as é a forma mais sólida de aprender através de uma postura crítica-criativa e consciente de suas responsabilidades. Por isso, a importância da prática assistencial nos serviços de saúde que oportunize uma aprendizagem ativa e reflexiva, além de permitir experiências motivacionais entre estudantes e docentes (COLLISELLI L, 2009; CAMELO $\mathrm{SHH}, 2012)$.

O Enfermeiro-docente inserido no trabalho em saúde, deve não somente manter-se atualizado, dentro de um processo educativo contínuo de ensino e aprendizagem que permeia desde as estratégias pedagógicas que vão além dos conteúdos desenvolvidos em sala de aula, como também, ele contribui como instrutor clínico dos estudantes, como mediador do aprendizado durante a prática do discente, através de uma boa congruência social e cognitiva que permita o desenvolvimento do aluno como um ser crítico-reflexivo-criativo, e consciente de sua responsabilidade ética, política e profissional. (BENITO GA; TRISTÃO KM, et al., 2012; FERREIRA FC, et al., 2017).

Assim, faz-se necessário que os professores utilizem estratégias que permitam conhecer e abordar as sensações vivenciadas pelos estudantes, tais como, dor, alegria, sofrimento, perdas, surpresas, dentre outras, oportunizando o dialogar, refletir, revisar, significar e modificar sua forma de vivenciar as situações enfrentadas, minimizando os danos causados pelo estresse, ou seja, atendendo as necessidades do estudante e colaborando para o seu desenvolvimento (ALVES EA, 2014).

Dessa forma, repensar no relacionamento docente - discente no campo de estágio é uma prioridade. Procura-se hoje em dia preparar futuros profissionais para a vida em equipe, tendo como requisitos, responsabilidade, empatia, preocupação com os clientes e colegas. Assim, é possível desenvolver a reflexão sobre o cuidado, não só na perspectiva do cliente, mas também de todos os envolvidos no processo da saúde/doença (SILVA RM, 2009; SILVA CM, 2013).

Diante da importância do estágio curricular para formação do profissional de enfermagem, sendo esse coberto de expectativas por parte dos acadêmicos, que esperam nesse período suprir as necessidades práticas para a efetivação da sua graduação, o objetivo desse estudo foi descrever as principais dificuldades e possibilidades vivenciadas pelos acadêmicos de enfermagem no exercício do estágio curricular supervisionado, correlacionando-as ao conhecimento didático pedagógico pré-formado com a rotina estabelecida nos serviços de saúde.

\section{METODOLOGIA}

Trata-se de uma revisão integrativa que permite incluir literatura teórica e empírica, além de estudos com diferentes desenhos metodológicos. Seu desfecho é a construção de uma conclusão a partir da síntese dos principais achados, possibilitando a análise do conhecimento preexistente sobre o tema investigado (MENDES KD, 2008).

Foi realizada ampla pesquisa de artigos publicados em periódicos CAPES/MEC, periódicos indexados nas bases da MEDICAL LITERATURE ANALYSIS AND RETRIEVAL SISTEM ON-LINE (MEDLINE), PUBMED, SCIENTIFIC ELECTRONIC IBRARY ONLINE (SCIELO), BIREME e Literatura Latino-Americana e do Caribe em Ciências da Saúde (LILACS), pela abrangência de artigos originais publicados em periódicos de área de enfermagem.

A seleção dos artigos foi efetuada nos meses de setembro 2018 a março 2019. Para a pesquisa foram utilizados primeiramente uma consulta aos descritores do DECS próprios das bases de dados LILACS, SCIELO e BIREME, no idioma português, inglês e espanhol, além de pesquisas nas diretrizes curriculares 
nacionais do Ministério da Educação do curso de graduação de enfermagem sobre estágios (Pareceres/Resoluções). Foram utilizados, para a busca dos artigos, os seguintes descritores: Educação Baseada em Competências, Educação em Enfermagem, Enfermagem e Estágio Clínico. Em seguida, foi necessário realizar a busca por descritores do MEDICAL SUBJECT HEADINGS (MESH) próprio a base de dados PUBMED E MEDLINE (MESH/TERMS).

Para obtenção das informações, durante a busca de dados foram obtidas as seguintes operações da pesquisa: Enfermagem AND Educação em enfermagem AND Estágio clínico; Enfermagem AND Estágio clínico AND Educação baseada em competências e Enfermagem AND Educação em Enfermagem AND Educação baseada em competências. Foi utilizado o Operador Booleano AND para formar o conjunto de descritores das equações dispostos na tabela 1, pois foi o único que mostrou uma busca precisa acerca do foco da temática, com isso foi possível encontrar os artigos para a elaboração dos resultados e discussão da pesquisa de forma rápida e precisa.

Tabela 1 - Estratégias de busca para consulta em bases de dados.

\begin{tabular}{|c|c|c|}
\hline Cruzamento em Inglês & Cruzamento em Espanhol & Cruzamento em Português \\
\hline $\begin{array}{ll}\text { Nursing (DECS) } & \text { AND }\end{array}$ & Enfermería (DECS) & Enfermagem \\
\hline Clerkship (MESH TERMS) & Educacion em Enfer & Enfermagem \\
\hline $\begin{array}{l}\text { Education, } \\
\text { TERMS). }\end{array}$ & $\begin{array}{l}\text { TERMS) AND Prácticas Clínicas } \\
\text { (MESH TERMS). }\end{array}$ & $\begin{array}{l}\text { (MESH TERMS) AND Estágio } \\
\text { Clínico (MESH TERMS). }\end{array}$ \\
\hline Nursing (DECS) & ECS) and Prácticas & Enfermagem (DECS) \\
\hline $\mathrm{p}$ (MESH TERMS) & 1S) and & Estágio Clínico (ME \\
\hline $\begin{array}{l}\text { רcy-Based } \\
\text { ERMS). }\end{array}$ & $\begin{array}{l}\text { Educación Basada en } \\
\text { Competencias (MESH TERMS). }\end{array}$ & $\begin{array}{l}\text { AND Educação B } \\
\text { Competências (MES }\end{array}$ \\
\hline $\begin{array}{l}\text { AND Educa } \\
\text { TERMS) }\end{array}$ & $\begin{array}{lr}\text { ECS) } & \text { AND } \\
\text { Enfermería (MESH }\end{array}$ & $\begin{array}{l}\text { Enfermagem (D) } \\
\text { Educação em }\end{array}$ \\
\hline $\begin{array}{l}\text { Competency-Based Education } \\
\text { (MESH TERMS). }\end{array}$ & $\begin{array}{l}\text { TERMS) and Educación Basada } \\
\text { en Competencias (MESH } \\
\text { TERMS). }\end{array}$ & $\begin{array}{l}\text { (MESH TERMS) AND Educação } \\
\text { Baseada em Competências } \\
\text { (MESH TERMS). }\end{array}$ \\
\hline
\end{tabular}

Fonte: LILACS, MEDLINE, BIREME, SCIELO E PUBMED.

Para a realização do estudo foram percorridas seis etapas: identificação do tema e seleção da questão de pesquisa; estabelecimento dos critérios de inclusão/exclusão (seleção da amostra); definição dos estudos pré-selecionados e selecionados; categorização dos estudos; análise e interpretação dos resultados; e apresentação da revisão/síntese do conhecimento.

Primeiro passo para o estabelecimento dos critérios de inclusão realizados pelos pesquisados e pelo consenso entre eles, se deu através da seleção manual e automática nas bases de indexação relacionados aos artigos originais, que estavam dentro do recorte temporal de 2016 a 2019, além dos artigos originais e relato de experiência, disponíveis na íntegra, que apresentaram uma abordaram quantitativa e qualitativa a temática - voltada as principais dificuldades vivenciadas pelos acadêmicos de enfermagem em relação as práticas exercidas no período de estágio supervisionado, sem restrição de idiomas, país de publicação. Sendo assim, os artigos foram classificados quanto à categoria do periódico, período de publicação, temática, tipo de pesquisa e instrumentos utilizados para análise.

Foram excluídos os artigos que fugiam da temática em questão, artigos que se apresentavam em duplicidade, artigos de opiniões sem base legal-científica, ponto de vista, carta ao leitor, editoriais que abordavam revisões de literatura, manuais técnicos, monografias, pesquisa sem nenhuma comprovação documental ou científica e artigos que fugiam do consenso entre os pesquisadores.

No que se refere à seleção automática, ela foi realizada de forma independente e em duplo-cego de títulos, resumos e textos completos, por dois membros da equipe de revisão, porém a medida que os pesquisadores 
encontraram diferenças em relação a quantidade e qualidade de estudos, foi realizada nova busca, respeitando-se com rigor, os critérios de inclusão e de exclusão.

E para a seleção manual realizada pelos pesquisadores e pelo consenso entre eles, os critérios foram: artigos originais publicados em periódicos e estudos relacionados a temática da pesquisa. Para execução dessa seleção manual foi realizada uma leitura aprofundada dos documentos a analisar e conhecer do texto. Nas divergências da seleção os autores recorreram ao pesquisador de maior experiência, como enfermeiro docente e assistencial.

\section{RESULTADOS}

Pelos resultados identificam-se inicialmente 123 trabalhos, destes 115 foram excluídos por ano de publicação. Para efetivação da busca em cada base de dados foram inseridos de forma combinada com o operador booleano Enfermagem AND Educação em enfermagem AND Estágio clínico; Enfermagem AND Estágio clínico AND Educação baseada em competências e Enfermagem AND Educação em Enfermagem AND Educação baseada em competências.

O processo de busca inicial foi feito pela aplicação dos descritores nas bases de produção científica, sem a utilização de nenhum critério de inclusão ou exclusão. Por este processo, deu-se início à aplicação de filtros onde foram identificados oito artigos selecionados por ano de publicação e com os descritores diretamente relacionados ao tema, respeitando os critérios de identificação, seleção, elegibilidade e inclusão das amostras quanti e qualitativas (Figura 1). Localizando-se, a partir desses, quatro estudos que atenderam aos critérios de inclusão e exclusão. Sendo definitivamente considerados para a produção da referida pesquisa.

Do total de produção científica (123), a base de dados LILACS sozinha apresentou 93 produções científicas, tendo mais produção que as bases de dados BIREME e SCIELO juntas. E a análise dos estudos selecionados, enfatizam a existência de uma lacuna entre a teoria e a prática no campo de estágio curricular supervisionado.

A Figura 1 apresenta o fluxograma da estratégia de seleção dos artigos, conforme os critérios de identificação, seleção, elegibilidade e inclusão pré-estabelecida.

Figura 1 - Fluxograma com estratégias de seleção dos artigos, após busca em base de dados eletrônicas, $2016-2019$.

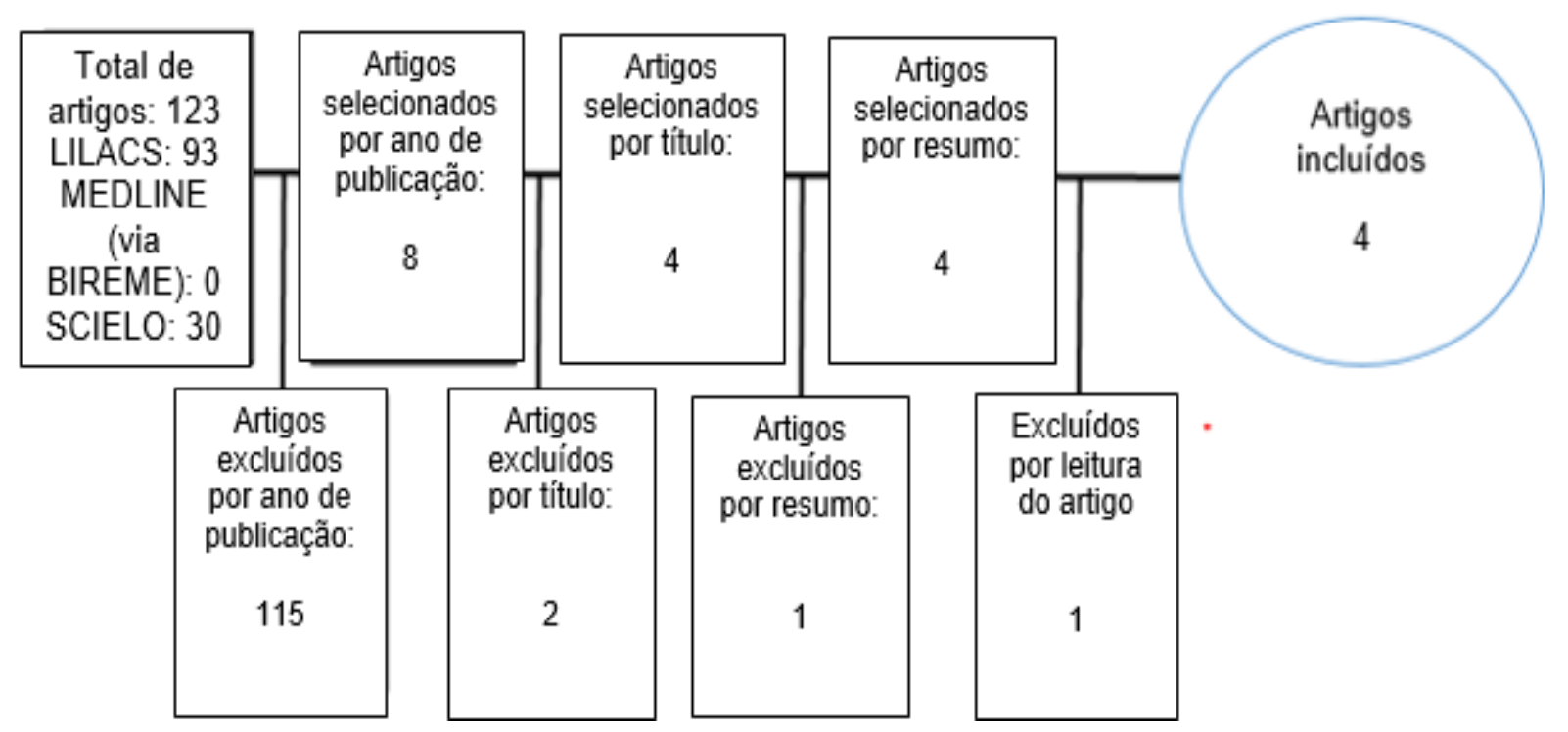

Fonte: Fluxograma construída pelos (as) autores (as) do projeto, utilizando como fonte as bases de dados: LILACS, MEDLINE, BIREME, SCIELO E PUBMED. 


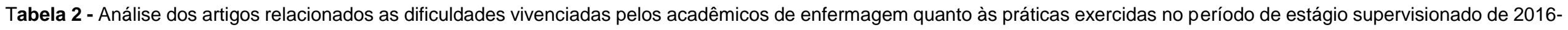
2019.

\begin{tabular}{|c|c|c|}
\hline Autor e Ano & Desenho do Estudo & Amostra \\
\hline $\begin{array}{l}\text { SALIFU DA; } \\
\text { SALIFU MA; } \\
\text { NINNONI JPK, } \\
2019\end{array}$ & $\begin{array}{l}\text { Metodologia descritiva } \\
\text { de abordagem qualitativa }\end{array}$ & $\begin{array}{l}\text { A amostragem de variação máxima } \\
\text { baseada no papel nos eventos da lacuna } \\
\text { entre teoria e prática foi usada para } \\
\text { recrutar enfermeiros estudantes, } \\
\text { enfermeiros docentes e clínicos de dois } \\
\text { locais de estudo para discussões em } \\
\text { grupos focais. Os dados foram analisados } \\
\text { utilizando análise de conteúdo } \\
\text { convencional }\end{array}$ \\
\hline $\begin{array}{l}\text { RIGOBELLO JL, et } \\
\text { al., } 2018\end{array}$ & $\begin{array}{l}\text { Questionário } \\
\text { AUTO-RELATÓRIO; } \\
\text { qualitativo } \\
\text { exploratório }\end{array}$ & $\begin{array}{l}87 \text { discentes (concluíram a disciplina do } \\
\text { ECS no primeiro semestre de } 2016 \text { ( } 63 \\
\text { alunos da IES estadual e } 24 \text { da IES } \\
\text { privada); } \\
280 \text { egressos formados nos anos } 2013 \text {, } \\
2014 \text { e } 2015 \text { ( } 195 \text { egressos da IES } \\
\text { estadual e } 85 \text { da IES privada) } \\
48 \text { docentes que atuaram por, no mínimo, } \\
\text { um semestre na disciplina do ECS, sendo } \\
42 \text { da IES estadual e } 6 \text { IES privada } \\
\text { Duas universidades paulistas. }\end{array}$ \\
\hline $\begin{array}{l}\text { MARCHIORO D; } \\
\text { CERATTO PC; }\end{array}$ & $\begin{array}{l}\text { Relato de } \\
\text { experiência }\end{array}$ & $\begin{array}{l}\text { Discentes de enfermagem que estão } \\
\text { cursando o } 10^{\circ} \text { Período }\end{array}$ \\
\hline
\end{tabular}

BITENCOURT JVO,

et al., 2017.

Objetivos

Descrever as experiências $e$

percepções da lacuna entre teoria

e prática em enfermagem em um

ambiente com recursos limitados

Esclarecer qual é a percepção dos egressos, concluintes e docentes acerca do processo de ensino-aprendizagem do Estágio Curricular Supervisionado dos Cursos de Graduação em Enfermagem e à luz do desenvolvimento das competências gerenciais descritas nas Diretrizes Curriculares Nacional.

Analisar a percepção de estudantes de enfermagem sobre o Estágio Curricular Supervisionado no seu processo de formação.

\section{Resultados}

Cinco temas foram identificados:

inadequações do sistema; restrições de

recursos; desafios do ambiente de

aprendizagem clínica; colocação clínica e supervisão; e enfermeira fatores da faculdade. A inadequação dos sistemas e as restrições de recursos formaram a espinha dorsal dos desafios que contribuíram para a lacuna entre teoria e prática.

Emergiram quatro categorias temáticas acerca desse processo:

O papel fundamental do enfermeiro supervisor no ECS.

A necessidade de maior presença dos docentes

Quanto às competências gerenciais, os sujeitos enfatizaram liderança, gestão de recursos e desempenho das funções burocráticas como essenciais.

A importância da adequada condução e realização do estágio supervisionado, por parte de professores, serviço e estudantes que objetivam participar ativamente do processo de formação do profissional enfermeiro.

Dessa forma, embora sejam reais e explícitos os conflitos vivenciados pelo estudante, durante o período de ECS é perceptível o quanto esta exposição do cotidiano do enfermeiro.

$54 \%$ dos estudantes relataram dificuldades Identificar a perspectiva dos quanto ao campo de estágio. Outras dificuldades foram a falta de prática, a resistências dos usuários, período curto de acadêmicos de enfermagem exploratório com abordagem quantitativa.

estágio e a pouca realização e

A maioria dos autores descrevem relevância dos estágios como uma oportunidade rica para o acadêmico colocar em prática todo seu poder de crítica, reflexão para tomada de decisão de acordo com a procedimentos.

Fonte: Tabela elaborada pelos (as) autores (as) do projeto, utilizando como fonte as bases de dados LILACS, MEDLINE, BIREME, SCIELO E PUBMED.

REAS/EJCH | Vol .Sup.18 | e662 | DOI: https://doi.org/10.25248/reas.e662.2019 Página 6 de 10 
A Tabela 2 foi construída para sintetizar os estudos, por meio das análises das seguintes variáveis: autor/ano; desenho do estudo; amostra; objetivos e resultados. De acordo com a classificação, as pesquisas enquadram-se como artigos originais $(n=3)$, e relato de experiência $(n=1)$, sendo os três de abordagem qualitativa e o último de abordagem quantitativa, porém os dois primeiros projetos apresentam desenhos metodológicos de formato idênticos entre eles, e os outros dois últimos apresentam características heterogênicas.

O primeiro artigo da tabela 2, fala sobre a ineficácia do sistema de preceptoria por falta de treinamento dos preceptores e dos múltiplos papéis assumidos por eles, além de relatar as baixas qualificações acadêmicas. E que a maioria das universidades no cenário de pesquisa está oferecendo agora programas de enfermagem de bacharelado sem qualquer roteiro claro para orientar o processo de integração do ensino de enfermagem nessas instituições de ensino superior. Enquanto, o segundo artigo de RIGOBELLO JL (2018), mostra o elo importante entre os docentes e os atores envolvidos, tais como, estudante, enfermeiro e profissionais dos serviços, de maneira a repercutir no sucesso ou insucesso do aprendizado do aluno no campo de estágio. O terceiro estudo (MARCHIORO D, 2017), enfatiza o papel do professor mediador, a articulação entre a universidade e o serviço de saúde e destaca o papel do ECS na formação dos discentes, com abordagem diferente ao do quarto artigo, que ressalta apenas as trajetórias na formação dos estudantes universitários, levando a uma complementariedade de informações.

\section{DISCUSSÃO}

Os artigos selecionados para a pesquisa enfatizam a necessidade de fortalecimento, não somente das estratégias de ensino, como também da integração da teoria com a prática entre os estudantes para o processo de aprendizagem (CORREA GC, 2017).

Essa etapa de fortalecimento voltado ao processo de ensino-aprendizagem possibilitou verificar que existem algumas lacunas abertas, uma delas são as instituições que utilizam como ferramenta pedagógica o ensino tradicional, tais como, competências que foram aprendidas somente por meio de conteúdo teórico durante as aulas e outras conseguiram ser aprendidas e previamente implementadas durante os estágios práticos, mesmo que de forma superficial, e somente aperfeiçoadas na prática hospitalar. Essas lacunas revelam que o futuro enfermeiro chega ao mercado com preparo limitado para enfrentar sua realidade de trabalho e, por isso, muitas vezes, buscam por capacitação e aperfeiçoamento profissional após a formação acadêmica (VIANA MA, 2016).

Já outros artigos enfatizam outros aspectos, a saber, a aquisição de conhecimento, nível de empatia, atitudes em relação aos outros, estados emocionais, prática reflexiva, autotranscendência, maturidade cognitiva, comportamentos de aprendizagem e perspectivas dos alunos, como elementos relevantes para a aplicação dos conteúdos teóricos na prática e que ainda não foram bem trabalhados. Para tanto, existe uma necessidade de acompanhar mudanças no quadro educacional (RIEGER CA, 2016). Verificou-se que apesar da Instituição de Ensino Superior (IES) selecionada a oferecer campo de estágio para que os alunos consigam desenvolver as competências que são exigidas no mercado de trabalho, os egressos de enfermagem apresentaram dificuldades quanto as habilidades aprendidas e desenvolvidas na graduação, e as que foram desenvolvidas e/ou implementadas no ambiente hospitalar.

Frente as deficiências percebidas ao longo dos anos nos projetos anteriores para os cursos tradicionais da área de saúde, fez-se necessário adotar um novo currículo integrado e orientado por competência dialógica, optando-se por dar continuidade à aplicação do Método da Aprendizagem Baseada em Problemas (ABP), na Unidade Educacional Sistematizada (UES), e do Método da Problematização, na Unidade de Prática Profissional Médica. Em 2005, o Ministério da Saúde, através do Programa Nacional de Reorientação da Formação Profissional em Saúde, reitera essa proposta de mudança curricular para todos os outros cursos de saúde (MS, 2009). Entende-se que essas modificações na estrutura educacional devem estar voltadas para um novo olhar do pedagógico, com ênfase ao desenvolvimento efetivo do espírito crítico, isto é, que permitam ao aluno desenvolver sua capacidade de questionar, investigar, divergir, argumentar, analisar, experimentar e avaliar a aplicabilidade das diversas teorias à prática. 
Em meio a tantas teorias educacionais e tendências pedagógicas que permeiam o processo de ensinoaprendizagem, especialmente na educação superior, esse estudo abre um parêntese importante quanto as dificuldades enfrentadas pelos discentes na aplicação do conteúdo teórico-prático durante os estágios curriculares supervisionados. Tais como, alinhamento entre docente e enfermeiro sobre atuação do aluno no setor, articulação do professor com o enfermeiro responsável no cenário de prática e maior integração da instituição de ensino não somente com o professor, mas também com o aluno estagiário e os serviços de saúde. Entende-se que esse processo de aprendizagem é de suma relevância, para que o objetivo do ECS seja concretizado em sua plena totalidade. O aprendizado baseado nesse contexto estimula 0 desenvolvimento de competências clínicas profissionais, além de melhorar a prestação dos cuidados de enfermagem de forma segura e de qualidade, através da contextualização dos conhecimentos adquiridos, voltados a uma prática dinâmica, inovadora e transformadora (COLLISELLI L, 2009; BENITO GA, 2012).

Um estudo semelhante foi desenvolvido por Quadros JS e Colomé JS (2016), onde enfatiza a importância do processo de articulação da teoria com a prática, utilizando novas metodologias de ensino-aprendizagem, que possibilitem mudanças nos sujeitos envolvidos nesse processo, tendo uma perspectiva de profissional crítico, reflexivo e compromissado com seu papel social. Outro aspecto salientado por COLLISELLI L (2009), foi que não se deve ver o estágio curricular como mero mecanismo de proporcionar ao aluno a aplicação da teoria aprendida em sala de aula, mas também, requer o desenvolvimento de habilidades e aplicação do conteúdo teórico à prática.

Para Lauder (2004) e Tuoriniemi P (2008), isso só podia ser alcançado a partir do momento em que os estudantes de enfermagem aplicassem o que aprenderam nos laboratórios de simulação e de sala de aula a situações do mundo real, garantindo assim, experiências cognitivas, psicomotoras e afetivas. Todos esses aspectos também foram confirmados em outros estudos (COVIZZI UD; SOBRAL FR, 2012; BARRETO DG, 2014), onde se propõe a necessidade de se garantir uma pedagogia efetiva e inovadora, por meio da simulação realística e finalizada com a discussão reflexiva (defriefing), como forma de fomentar o processo educativo.

Entretanto, vivemos em uma época de rápidas e profundas transformações, as quais impõem novas formas de pensar e agir que exigem um processo de formação estimulador da capacidade de entender como se produz o saber nas diversas áreas. Diante deste cenário, Torres e colaboradores (2015), observaram o quanto se faz necessário a aplicação de metodologias que estabeleçam a mediação entre o conhecimento e o aluno de forma inovadora, visando a solidificação e ampliação do processo-aprendizagem.

A prática docente do novo milênio demanda, cada vez mais, a busca por novas alternativas metodológicas de ensino. Do professor, portanto, é exigido que além do conteúdo do programa de ensino, oportunize aos discentes outras formas de aprendizagem mais interativas e que permitam a assimilação de conhecimentos, tendo em vista que as dinâmicas da contemporaneidade estabelecem relações intersubjetivas de maneira heterogêneas (TORRES, 2015).

Sobre outros aspectos relacionados à atuação do professor, Rigobello JL (2018) enfatiza a baixa atuação dos preceptores em relação as particularidades individuais apresentadas por cada aluno, posto que no contraditório, os acadêmicos sentir-se-iam mais amparados quanto ao esclarecimento de dúvidas e/ou questionamentos, embora há controversas em relação ao docente ser resolutivo no campo de estágio, porque isso pode gerar limitações quanto ao desenvolvimento dos acadêmicos, pois para Marchioro D (2017), no que tange ao papel do próprio ECS, o processo de formação, possibilita a expressão de fragilidades e potencialidades dos estudantes, por meio de erros e acertos no desenvolvimento das atividades diárias, visto que configura possibilidade de aprendizado, tanto na presença do enfermeiro supervisor e professor mediador, quanto na ausência desses. No entanto, no que se refere aos erros no desenvolvimento das atividades realizadas pelos discentes em ECS, isso pode ser uma ferramenta perigosa e principalmente quando se trabalha com paciente real na ausência do professor-preceptor, para isso Sanino GE (2012), faz uma ressalva para a oportunidade de se desenvolver simulações realísticas em ambiente controlado e protegido, antes do aluno ingressar ao campo de estágio e com isso gerar autonomia no cuidado, raciocínio rápido e intervenções adequadas. 
Rigobello JL (2018) et al, explicam que a intervenção do docente supervisor é fundamental no ECS. Ele é o responsável por preparar o campo de estágio apropriado para os estudantes desempenhar suas funções com segurança, orientar os funcionários e alunos visando uma melhor interação entre ambos, estar presente em tempo oportuno, enquanto durar o estágio, observar e detectar momentos conflituosos, ser experiente para fomentar o desempenho acadêmico, além de ser considerado um ponto de intercessão entre a universidade, aluno e local de estágio.

Uma pesquisa semelhante realizada por Marchioro D et al, (2017), quanto às atribuições do professor, identificam que é preciso definição clara de suas funções como articulador entre instituição de ensino e serviço. O docente deve em conjunto com universidade/serviço e estudante elaborar propostas pedagógicas para o ECS, definindo objetivos, métodos e intervenções exequíveis no encontro das Diretrizes Curriculares Nacionais e do perfil do egresso. Marchioro D (2017) relata em sua pesquisa que, cabe ao professor facilitar o desenvolvimento do ECS, em especial na fase introdutória do estudante no cenário da prática, aproximandoo do enfermeiro responsável pela unidade e esclarecendo seu papel no desenvolvimento deste componente, para que se evite o choque de realidade, exposto por Al Awaisi H e Pryjmachuk S (2015), em que considera a existência de uma lacuna entre a teoria e a prática, como uma das principais causas da baixa satisfação no trabalho e das altas taxas de atrito entre o preceptor de campo de estágio e os enfermeiros recém-formados.

Outro trabalho de Rigobello JL (2018), enfatiza a baixa proximidade do docente-preceptor ao grupo de alunos estagiários, posto que várias dificuldades vivenciadas no decorrer do ECS poderiam ter sido amenizadas ou evitadas, tais como, sentimentos de incapacidades, frustração, passividade, visto que, a aproximação docente-discente, traria mais segurança e tranquilidade aos acadêmicos quanto ao seu desempenho e atividade pré-profissional. Dados esses, que se assemelham aos encontrados na pesquisa de Rodrigues (2011), em que considera necessário rever o relacionamento entre preceptores, estagiários e professores no decorrer do estágio, de forma que os preceptores tenham um papel de mediador e facilitador para a execução de uma prática pedagógica centrada no aluno.

Dentro dessas perspectivas, vimos que o acadêmico de enfermagem enfrenta inúmeros desafios durante o estágio curricular supervisionado, dentre eles podemos destacar: relacionamento aluno-preceptor, baixa capacitação técnica e psicológica dos professores, poucas metodologias acolhedoras para atender as dificuldades individuais dos alunos, pouca presença junto ao aluno durante a execução das práticas assistenciais, fatores esses que acabam implicando negativamente no desenvolvimento de competências teórico-práticas; e no cumprimento efetivo do papel do ECS na vida do acadêmico de enfermagem.

Entretanto, formar pessoas é algo que demanda bastante empenho do profissional docente que por sua vez está inserido dentro de estabelecimentos de saúde - que muitas vezes não lhe dão o aporte necessário para promover o conhecimento entre os acadêmicos - onde deveria ocorrer o contrário, considerando que a preocupação maior deveria ser a formação do aluno enquanto ser humano, já que o ambiente acadêmico promove inúmeras teorias, mas que não há ainda práticas capazes de implementar as mudanças essenciais na formação desses profissionais.

\section{CONCLUSÃO}

O Estágio Curricular Supervisionado tem um papel fundamental para o discente de enfermagem por ser o momento onde visa o desenvolvimento e aprimoramentos das competências teórico-práticas; onde se chega mais próximo do que se espera pós conclusão do curso. Momento esse que deveria lhe dar todo o aporte profissional para execução das tarefas assistências de enfermagem, tomada de decisões, gerenciamento de pessoas e conflitos. Embora seja visível os desafios enfrentados pelos acadêmicos de enfermagem, esse estudo mostra a necessidade de integração das disciplinas teórico-prática e da articulação entre instituições de ensino e serviços de saúde para que, de fato, o princípio da integralidade seja incorporado na formação dos (as) acadêmicos de enfermagem. Posto que esse momento é promissor de experiências profissionais, as quais visam fortalecer o processo de ensino-aprendizagem, além de proporcionar uma visão mais realística sobre a sua atuação como futuros enfermeiros. 


\section{REFERÊNCIAS}

1. AI AWAISI H, et al. The experiences of newly graduated nurses during their first year of practice in Sultanate of Oman: A case study. International Journal of Nursing Studies, 2015; 52, 1723-1734

2. ALVES EATD, COGO ALP. Percepção de estudantes de enfermagem sobre o processo de aprendizagem em ambiente hospitalar, Revista Gaúcha Enfermagem, 2014; 35(1):102-109.

3. BARRETO DG, et al. Simulação realística como estratégia de ensino para o curso de graduação em enfermagem: Revisão Integrativa. Revista Baiana de Enfermagem, 2014; 28(2):208-14.

4. BENITO GAV, et al. Desenvolvimento de competências gerais durante o estágio supervisionado. Revista Brasileira de Enfermagem, 2012; 65(1): 172-8.

5. COLLISELLI L, et al. Estágio curricular supervisionado: visionado: diversificando cenários e fortalecendo a interação ensinoserviço. Revista Brasileira de Enfermagem, 2009; 62(6): 932-7.

6. CORREA GC. Definição e desenvolvimento de competências: um paradigma no processo estratégico. Revista do CEPE, 2015; 39(67):103-16.

7. COVIZZI UDS, LOPES DE ANDRADE PF. Estratégia para o ensino do metabolismo dos carboidratos para o curso de farmácia, utilizando metodologia ativa de ensino. Revista Brasileira de Ensino de Bioquímica e Biologia Molecular, 2012; v. 39, n. 67, p. 103-116.

8. CUNNINGHAN TMP, et al. O novo professor do curso técnico de enfermagem, num contexto atual da educação: uma visão crítico-reflexiva. Revista Técnicocientífica de Enfermagem, 2003; p.353.

9. EVANGELISTA DL, IVO OP. Contribuições do estágio supervisionado para a formação do profissional de enfermagem: expectativas e desafios. Revista Enfermagem Contemporânea, 2014; 3(2):123-30.

10. FERREIRA FDC, et al. Saberes e competências do enfermeiro para preceptoria em unidade básica de saúde. Revista Brasileira de Enfermagem, 2018; 71(suppl 4):1657-65.

11. KEISER DE, SERBIM AK. Diretrizes curriculares nacionais: percepções de acadêmicos sobre a sua formação em enfermagem. Revista Gaúcha Enfermagem, 2009; 30(4):633-40.

12. LIMA TC, et al. Supervised curricular internship: analysis of the students' experience. Revista Brasileira de Enfermagem, 2014; 67(1):133-40.

13. MARCHIORO D, et al. Estágio curricular supervisionado: relato dos desafios encontrados pelos (as) estudantes. Arq Ciênc Saúde UNIPAR [Internet]. Arquivos de Ciências da Saúde da UNIPAR, 2017; 21(2):119-22.

14. MENDES KDS, et al. Revisão integrativa: método de pesquisa para a incorporação de evidências na saúde e na enfermagem. Revista Texto Contexto Enfermagem, 2008; 17(4): 758-64.

15. MINISTÉRIO DA EDUCAÇÃO (BR). Conselho Nacional de Educação. Resolução № 3, de 07 de novembro de 2001. Institui Diretrizes Curriculares Nacionais do Curso de Graduação em Enfermagem. 2001 [cited 2017 Aug 18]. Disponível em: http://www. content/uploads/2012/03/resolucao.CNE.CES_3_2001Diretrizes_Nacionais_Curso_Graduacao_Enfermagem.

16. MINISTÉRIO DA SAÚDE E EDUCAÇÃO. PROGRAMA NACIONAL DE REORIENTAÇÃO DA FORMAÇÃO PROFISSIONAL EM SAÚDE - PRÓ-SAÚDE: OBJETIVOS, IMPLEMENTAÇÃO E DESENVOLVIMENTO POTENCIAL / Ministério da Saúde, Ministério da Educação. - Brasília: Ministério da Saúde, 2009.

17. QUADROS JS, COLOMÉ JS. Metodologias de ensino-aprendizagem na formação do enfermeiro. Revista Baiana de Enfermagem, Salvador, 2016; v. 30, n. 2, p. 1-10.

18. RIGOBELLO JL, et al. Estágio Supervisionado e competências gerenciais. Escola Anna Nery, 2018; 22(2).

19. SALIFU DA, et al. Experiences and perceptions of the theory-practice gap in nursing in a resource-constrained setting: $A$ qualitative description study. Nurs Open, 2019; 6(1): 72-83.

20. SANINO GE. O uso da simulação em enfermagem no Curso Técnico de Enfermagem. J Health Inform, 2012; 4(nesp):14851.

21. SILVA CMV, et al. Sentimentos dos enfermeirandos frente ao estágio curricular: quais as dificuldades e expectativas? Cadernos de Graduação - Ciências Biológicas e da Saúde Facipe, Recife, 2013; v. 1, n.1, p. 51-66.

22. SILVA, RM, et al. Ensino de enfermagem: reflexões sobre o estágio curricular supervisionado. Revista Práxis, 2009; ano I, $\mathrm{n}^{0} 1$.

23. SOBRAL FR, CAMPOS CJG. Utilização de metodologia ativa no ensino e assistência de enfermagem na produção nacional: revisão integrativa. Revista da Escola de Enfermagem da USP, 2012; v. 46, p. 208-218.

24. SOUZA FA, PAIANO M. Desafios e dificuldades enfrentadas pelos profissionais de enfermagem em início de carreira. REME - Revista Mineira de Enfermagem, 2011; 15(2): 267-273.

25. TORRES EB, et al. Aplicando princípios de gamificação em jogo educacional para apoiar o turismo educativo na cidade de Garanhuns. JORNADA DE ENSINO, PESQUISA E EXTENSÃO DA UFRPE, Recife, Anais Eletrônicos, 2015.

26. TUORINIEMI P, SCHOTT-BAER D. Implementing a highfidelity simulation program in a community college setting. Nurs. Educ. Perspect., 2008; v.29, n.2, p.105-9.

27. VIANA MAS, et al. Analysis of attitudinal assessment in the education of mid-level nursing professionals. Revista Eletrônica de Enfermagem, 2016; 18:1-10. 